\title{
Capsule Commentary on Turner et al., Chronic Opioid Therapy Urine Drug Testing in Primary Care: Prevalence and Predictors of Aberrant Results
}

\author{
Joseph Chiovaro, MD \\ Oregon Health and Science University, Portland VA Medical Center, Portland, OR, USA.
}

J Gen Intern Med 30(2):241

DOI: $10.1007 / \mathrm{s} 11606-014-3091-7$

(c) Society of General Internal Medicine 2014

$\mathrm{O}$ ne of the great public health challenges in this country is the use and misuse of opioid pain medications. Drug overdose deaths now outnumber deaths due to motor vehicle accidents, and a growing proportion of these deaths are due to opioid medications. ${ }^{1,2}$ The use of urine drug screening (UDS) to monitor compliance and to detect the use of other substances is a part of many opioid treatment programs. However, guidelines vary in their recommendations for UDS use, ${ }^{3}$ and surprisingly little is known about risk factors for aberrant results.

Turner and colleagues have added an important study to inform this discussion. ${ }^{4}$ Among primary care patients who underwent UDS while enrolled in a chronic opioid therapy program at an integrated group practice, $30 \%$ had aberrant results, including $12.3 \%$ for non-detection of the prescribed opiate. This is a group of particular concern, as opioid nondetection can be a marker of possible diversion. The chance of any aberrancy was higher in males, younger patients, those with a prior substance use disorder, and current smokers - all risk factors for opioid misuse. Importantly, the study authors further categorized the risk factors for each different reason for aberrant UDS result and found significant variation. For example, the presence of THC was more common in males, while other illicit drug identification was not. Lack of opiate detection in urine was more common among patients less than 45 years of age and with prior substance abuse disorders.
This topic reveals the intersection of epidemiology and stereotype in medicine. On the one hand, there are groups of patients who have statistically higher rates of aberrant UDS results, and applying a targeted testing policy to these patients could be supported as a potentially cost-effective way to monitor those at highest risk of misuse. On the other hand, given the stigma surrounding opioid monitoring, selective testing based on demographics has the real potential to negatively affect provider-patient relationships and to ostracize patients who might otherwise benefit from these medications. While risk factors for aberrant test results can't be ignored, they are probably best left as informative characteristics rather than fundamentals of policy, and it seems prudent to test uniformly, regardless of demographics.

Conflict of interest: The author declares that he has no a conflict of interest.

Corresponding Author: Joseph Chiovaro, MD; Oregon Health and Science University, Portland VA Medical Center, Portland, OR 97239, USA (e-mail: jchiovaro@gmail.com).

\section{REFERENCES}

1. Centers for Disease Control and Prevention. Addressing prescription drug abuse in the United States: Current activities and future opportunities. Department of Health and Human Services, Centers for Disease Control and Prevention 2013. Available at http://www.cdc.gov/HomeandRecreationalSafety/pdf/ HHS_Prescription_Drug_Abuse_Report_09.2013.pdf.

2. Centers for Disease Control and Prevention. WONDER [database]. Atlanta, GA: US Department of Health and Human Services, Centers for Disease Control and Prevention; 2013. Available at http://wonder.cdc.gov.

3. Nuckols TK, et al. Opioid prescribing: a systematic review and critical appraisal of guidelines for chronic pain. Ann Intern Med. 2014;160:38-47.

4. Turner JA, et al. Chronic opioid therapy urine drug testing in primary care: prevalence and predictors of aberrant results. JGIM (DOI: 10.1007/s11606014-3010-y)

Published online November 12, 2014 